\title{
The Effectiveness of Integrating Educational Robotic Activities into Higher Education Computer Science Curricula: A Case Study in a Developing Country
}

\author{
Ernest B.B. Gyebi, Marc Hanheide, and Grzegorz Cielniak \\ School of Computer Science \\ University of Lincoln \\ LN6 7TS, Lincoln, UK \\ \{ergyebi, mhanheide, gcielniak\}@lincoln.ac.uk
}

\begin{abstract}
In this paper, we present a case study to investigate the effects of educational robotics on a formal undergraduate Computer Science education in a developing country. The key contributions of this paper include a longitudinal study design, spanning the whole duration of one taught course, and its focus on continually assessing the effectiveness and the impact of robotic-based exercises. The study assessed the students' motivation, engagement and level of understanding in learning general computer programming. The survey results indicate that there are benefits which can be gained from such activities and educational robotics is a promising tool in developing engaging study curricula. We hope that our experience from this study together with the free materials and data available for download will be beneficial to other practitioners working with educational robotics in different parts of the world.
\end{abstract}

Keywords: educational robotics, effectiveness of teaching methods, education in developing countries, tertiary education

\section{Introduction}

There is a general belief that in learning, robots can be an effective means to facilitate more engagement, higher motivation, and the development of practical skill sets, beyond the focus of robotics itself. For instance, the effectiveness of robotics as a subject to convey a larger skill sets to students has been analyzed and found to be promising [1]. The positive effect, to a large extent is gained from the "embodiment" and physical presence of robots, which make the outcomes of programming very vivid and immediately accessible, providing a continual formative assessment of learning progress and encouragement to students. Following these ideas, selected institutions in developing countries have recently made some efforts to break the traditionally very theoretical and presentation-based lecturing and introduced robotic activities to improve the quality of teaching and learning [2]. However, most of the studies in this area 
so far involved recreational and extracurricular activities, which do not provide an environment for assessing its effectiveness for teaching formal courses. In the study presented in this paper, we set out to embed constructionist learning by means of robot programming into an existing formal classroom curriculum in a developing country to test its effectiveness and impact. Particularly, in this paper, we present a study to investigate the effects of educational robotics on an undergraduate Computer Science education which was carried out in a public university in Ghana. In this work, robotics itself is not among the key learning objectives, rather is it employed as an educational tool set to help conveying key concepts of computing in the first year of an undergraduate degree programme.

The key contributions of this paper are the longitudinal design of the study, spanning the whole duration of one taught course, and its focus on continually assessing the effectiveness and the impact of robotic-based exercises as opposed to paper-based exercises. The study assessed the students' motivation, engagement and level of understanding in learning general computer programming. In particular, we set out to answer the following three research questions:

(i) Does learning with educational robots have impact on students' understanding of programming concepts?

(ii) Does interacting with educational robots have impact on students' motivation and engagement in learning programming?

(iii) Is the use of robotic set-up as a tool for conducting educational activities effective?

To answer these questions and determine the impact of educational robotics on students' learning, a 10-week full semester study involving educational activities was conducted at the Department of Computer Science, University of Ghana. The activities were embedded into one of the relevant courses on computer architecture and principles of programming. Students' responses to their interactions with the learning activities as well as examination scores from five pre-intervention years and the intervention year were analyzed for trends in students' performances.

\section{Related Work}

Effective teaching and learning in higher education is one of the biggest challenges faced by many developing countries. For example, the curricula of most institutions in these countries are usually designed to encourage the traditional lecture-based method of teaching without instructional strategies that facilitate the inclusion of all students [3]. Lecturers tend to use the old-fashioned style of teaching whereby emphasis is placed on information transmission rather than on students active involvement. This invariably reduces students engagement and participation in the lessons. Also due to the introduction of measures like affirmative action aimed at improving the representation of disadvantaged groups on the campuses, which is perhaps peculiar to developing countries [4], categories of students enter the universities with gaps in the knowledge and skills required for studying particularly in key areas such as mathematics and science [5]. Con- 
sequently, many of these students may be under-prepared to undertake university tasks which usually present challenges for students to overcome. Therefore selected institutions in developing countries have recently made efforts to introduce effective ways of improving the quality of teaching and learning in order to support the students.

One of the practices that has been identified particularly, in computer science, and is promoted by many of the western higher education institutions [6] to effectively improve teaching and learning involves hands-on exercises and collaborative learning. Some educators such as T.M. Akey also argue that students learn more and retain more information when they actively participate in the learning process and when they can relate to what is being taught [7]. Therefore efforts to improve the quality of teaching and learning by the adoption of practices that facilitate more engagement, higher motivation and active participation of students are a promising direction.

Educational theorists such as S. Papert [8] believe that robotic activities have tremendous potential to improve classroom teaching. They argue that students can gain a sense of power over technology by creating an environment where they can program computers and robots. Other studies have also identified the concrete nature of robots as being one of their important advantages when used as learning tool. For example, students can understand abstract concepts and gain a more functional level of understanding when they learn with robots [9]. However, it is important to emphasize that the robot is just another tool, and it is the educational theory that will determine the learning impact coming from robotic applications. Alignment with theories of learning, proper educational philosophy, well designed curricula and supportive learning environments are some of the important elements that can make any educational innovation, including robotics, successful [10].

Activities with educational robotics can serve learning objectives from a wide range of disciplines from technology and design to mathematics and science education. They are hands-on activities with important experimentation features [11]. From this point of view educational robotics creates an active, cooperative learning environment which emphasizes on students' participation. So incorporating robotic technologies into tertiary curriculum can enrich teaching practices with great impact in addressing teaching objectives from different disciplines with an innovative approach. This fact is backed up by research which suggests that robots tie into a variety of disciplines [12]. A robot is made of component parts of motors, sensors and software. Each of these parts depends on different fields of knowledge such as engineering, electronics, and computer science. This interdisciplinary nature of robots means that when students learn to engineer robots they will inevitably learn about the many other disciplines that robotics utilize [13].

Accordingly, educational robots are being used to teach various subjects at the university level. At Carnegie Mellon University, USA, an open source robot application development framework called Tekkotsu has been designed specifically for education [14]. This application development framework is based on 
$\mathrm{C}++$ which can be used for teaching maths topics such as vectors, matrices and linear algebra. Educational robotics has also been successfully used to teach Physics for undergraduate students in Brazil [15]. Students developed prototype robots that they used to learn electricity and electronics, with emphasis on building electrical circuits. In their study at the Department of Computer Science, University of Waterloo, B.W. Becker [16] also used 'karel' the robot for teaching Java programming. They designed an Introductory to Computer Science course that used the robot to teach object oriented approach to programming.

Despite these encouraging reports, the majority of considered studies are short-term, conducted for a selected subject and group of students only. In our previous work [17] we presented initial results from implementing small scale pilot studies on introducing educational robotics into higher education activities in developing countries. The current work takes these efforts one step further and reports on fully integrated educational robotic activities into undergraduate Computer Science curricula in an attempt to address some of the challenges of higher education in developing countries.

\section{Methodology}

The purpose of this study was to assess the impact of educational robotics on a formal undergraduate Computer Science education in developing countries. Our goal was to determine the effectiveness of educational robotics activities on students' motivation, engagement and level of understanding in learning computer programming. In this section, we present the methodological considerations and procedures adopted for the study, which include the course overview, learning activities, study design, surveys and participants.

\subsection{Course overview}

Our study considered a core Introduction to Computer Science course teaching basics of computer architectures and programming principles to all first year students at the Department of Computer Science, University of Ghana. The course consists of lectures and laboratory workshops, and its syllabus includes introduction to programming languages, data representation, logic operations but also program structure and flow control (see Sec. 3.2). In a typical setting, the lab workshops involve paper-based activities designed to enable students practice the topics learned in lectures. The structure of the paper-based activities is based on collaborative learning, an instructional method in which students work together in small groups toward a common goal [18]. Team work is emphasized and the students are assessed as a group. Therefore each student has a shared responsibility for meeting the course challenges such as submitting exercises for marking on time. The designed learning activities are usually in a form of handson exercises which often require computers in the lab. Students do the exercises on paper and submit their work to teaching assistants who also provide in-class help in case of difficulties. 


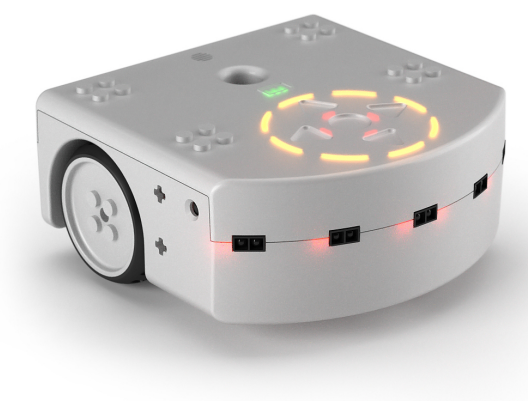

(a) Thymio II educational robot.

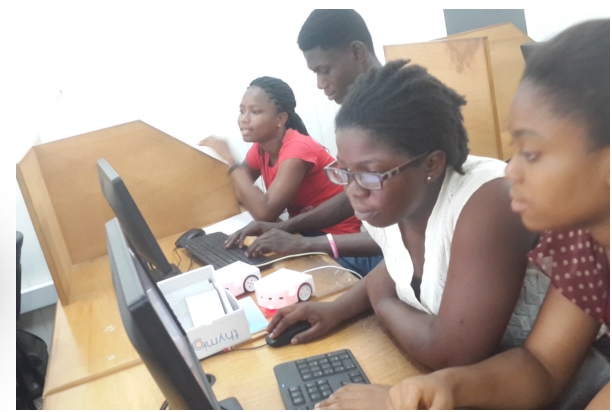

(b) Our students taking part in robotic activities in the lab.

Fig. 1: Lab activities using educational robots.

Our focus was to embed robotic activities alongside the paper-based activities in order to make direct comparisons between the two. Based on our earlier survey [19] which looked at the existing suitable robotic platforms for teaching Computer Science at African institutions, we have selected an affordable robot Thymio II to conduct the robot-based activities. Thymio II (see Figure 1 (a)) is a simple two-wheel differential platform equipped with an array of LEDs and a number of simple sensors including odometry, proximity and temperature sensors, a microphone, etc. The robot comes with a dedicated programming environment called Aseba, which is an open-source scripting language, and a set of on-line tutorials and additional materials that can be used when designing own teaching activities. We build on top of that setup by introducing a set of workshops thematically covering the five computer science topics described in the following sections. We share the developed materials including workshop descriptions, survey questionnaires and the course module in an open-source fashion on our website http://lncn. eu/gyebi16edu so these can be re-used by other educators teaching similar topics.

\subsection{Learning activities}

Our designed learning activities comprise of five workshops, all geared towards assisting students to learn the basic concepts in programming introduced during lectures. The course description and specific learning outcomes that students were expected to achieve at the end of each workshop are presented in Table1, which was adopted from existing course content and learning outcomes for the core introduction to Computer Science course. We have made it available on our website. We used an iterative, incremental development approach involving intermediate constructs, each adding more capabilities to make the tasks cognitively appropriate to the developmental levels of our students. Students were gradually introduced to the learning activities by performing the easier tasks before progressing to more difficult tasks which required application of skills and 
knowledge acquired from previous activities. To enable a direct comparison between paper- and robot-based activities, we created a set of similar tasks based on the same design goal for each of the five workshops, which we now describe in detail.

Table 1: Introduction to Computer Science - course description and associated learning outcomes.

\begin{tabular}{|l|l|}
\hline Topic & Learning Outcomes \\
\hline $\begin{array}{l}\text { Introduction to programming languages, } \\
\text { modern standards and applications }\end{array}$ & $\begin{array}{l}\text { Identify the various types of program- } \\
\text { ming languages and their use }\end{array}$ \\
\hline Data representation and number systems & $\begin{array}{l}\text { Understand representations for different } \\
\text { data types, perform conversions between } \\
\text { different number systems }\end{array}$ \\
\hline Digital logic and Boolean algebra & $\begin{array}{l}\text { Implement logic operations using the ba- } \\
\text { sic types of logic gates }\end{array}$ \\
\hline $\begin{array}{l}\text { Basic data types, operators and program } \\
\text { structure }\end{array}$ & $\begin{array}{l}\text { Demonstrate the ability to use variables, } \\
\text { operators and functions }\end{array}$ \\
\hline $\begin{array}{l}\text { Program control structures, branching } \\
\text { and loops }\end{array}$ & $\begin{array}{l}\text { Apply control structures to write simple } \\
\text { programs }\end{array}$ \\
\hline
\end{tabular}

Introduction to programming languages: The learning activities introduced students to the main types of programming languages and how they are used to write a sequence of instructions that a computer can execute. Since most of the students were learning programming for the first time, the challenge was how to assist them in building simple programs. Therefore, the paper-based activities concentrated on getting familiar with the programming environment through building provided examples in Visual Basic and teaching how to make simple code modifications, commenting and debugging. In the first week of robotbased activities, the students were introduced to the robot, its components and Aseba programming environment. Similarly to the paper-based activities, the students were provided with simple demo programs which illustrated individual parts of the setup and basics of program execution. These activities were mainly based on the existing tutorials for the Thymio robot.

Data representation: This workshop was designed to teach students practical aspects of number systems and data representation. Paper-based tasks required the students to exercise number system conversions from decimal to binary using the repeated division method. Difficulty of the task depended on the value of the converted numbers. Further tasks included also manual conversions into one's and two's complement representations. For the robot-based activities, we adopted the robot's 8 LEDs as a way of visualizing binary numbers and using each LED to represent a single bit. This way the students could visually see 
the results of counting in unary and binary systems, conversions from decimal to binary and different complement representations. The conversion code was provided and students were only required to make small changes to the code (e.g. changing variable values). Conversions from binary to decimal number system proved to be more difficult to implement as there were no obvious ways of visualizing the decimal results either on the robot or in software and therefore these activities were not included.

Logic Operations: The learning activities were designed to teach fundamentals of Boolean algebra, basic logic gates and a way of combining them into more complex systems. The paper-based activities included a range of standard exercises involving drawing gates and circuits and deriving truth tables. For the robot-based activities, we used proximity sensors as inputs to logic circuits so the robot movement was depended on logical combination of these inputs. This enabled learning about fundamental logic operations, universal logic gates but also Boolean functions combining multiple inputs through different operators. This final task required students to combine the provided code for individual logic gates into a combinatorial circuit and so teaching them how to reuse and restructure the existing code.

Programming Fundamentals: This workshop introduced students to the programming fundamentals including data types, variables, operators and functions. The paper-based tasks required association of different data types with variables, changing mathematical expressions into simple programming statements and also using programming operators to represent given statements. For the robot-based activities, the concept of variables was re-iterated by using timers and speed variables with basic movement behaviors. The students could observe differences in robot behavior depending on different values of these variables. The analysis of the provided code examples enabled students to learn about different types of operators. Further tasks required combining simple behaviors implemented as functions into more complex behaviors such as driving in a square. Due to certain limitations of the Aseba language, function parameters are not easily implemented and therefore were not exercised in the proposed activities.

Control Structures: The final set of workshop activities was designed to introduce program control structures such as conditional statements and loops. For the paper-based activities, programming tasks involved writing simple algorithms that would satisfy certain conditions by using IF-ELSE statements and tasks involving drawing of flow charts and implementing loops for algorithms calculating average values. Robot-based activities involved simple obstacle avoidance behaviors using conditional statements controlling the robot movement depending on the state of proximity sensors or controlling the direction of movement depending on manual key presses. The loops and conditional statements were employed to check the state of an array of 7 proximity sensors which then defined different robot movement. 


\subsection{Study Design}

In contrast to our earlier study [17] which was conducted as a relatively short pilot to identify the main implementation issues, the proposed activities were incorporated into a regular program of study and formed part of the learning activities for the first year undergraduate curricula. The experimental design used one group made up of all first year Computer Science students, but the learning activity was divided into two parts (referred to as paper-based and robot-based) to allow for direct comparison of the experimental results. The ethical policy of the University of Ghana required that students in a particular year group learn the same course topics and content in all formal courses in order to give the best and similar

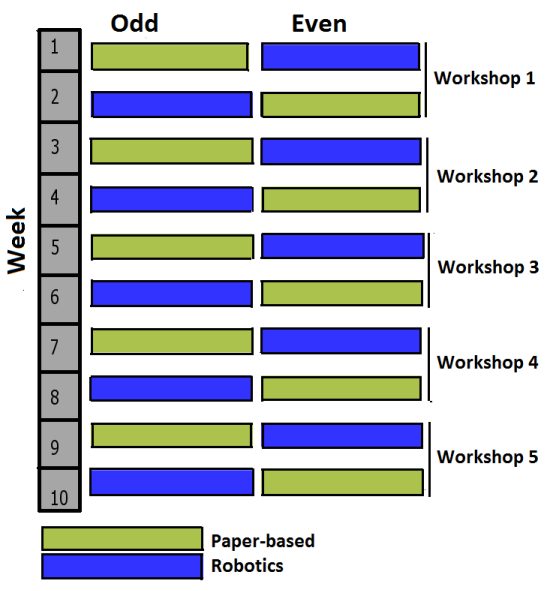

Fig. 2: Weekly schedule for workshop activities. experience to all students. Also there were resource constraints such as inadequate labs which posed organizational problems of systematic issues with timetabling of the sessions, preventing us from splitting the class into two distinct sets in a formal setting. As a result, we could not implement an experiment with a typical control group in our situation.

Each of the five workshop activities consisted of paper-based and robot-based tasks, which required 2 sessions to complete. The students were divided into 10 groups and those in odd numbered groups (i.e., 1, 3, 5, 7 and 9) participated in the paper-based activities while the remaining even numbered groups $(2,4,6,8$ and 10) practiced the robotic activities, initially. The groups were swapped for the odd and even numbered groups to participate in the robot-based and paperbased activities respectively in the following workshop session to complete the first workshop activity. This was repeated for all five sessions and therefore all students participated in all workshop activities. Figure 2 depicts the workshop schedule in a graphical format.

\subsection{Student Surveys and Performance}

Student surveys which included an initial survey, workshop evaluations and a final survey were conducted through a set of questionnaires to determine the student's motivation, engagement and level of understanding in both the paperbased and robotic activities. The purpose of the initial survey was to gain a better understanding of student background and knowledge before the study. The final survey provided means to evaluate the overall workshop experience and 
knowledge gained, whereas workshop evaluations helped assess the effectiveness of each lab session. The specific questions for respective surveys were grouped into following categories:

- Initial Survey asked for student background such as prior education, gender, selected study course but also prior learning experience in programming and using robots.

- Workshop Evaluation was issued to students after each session for both paper-based and robot-based activities. To encourage regular participation and honest answers, we made this part completely anonymous and consisting only of three questions on workshop difficulty, relevance to concepts learnt from lectures and re-use of concepts learnt in previous sessions.

- Final Survey included overall ratings for both paper-based and robot-based activities with regard to student motivation and engagement, intuitiveness and understanding. To gain the learning experience, the students were also asked about topics learnt and if they perceived them as beneficial. Finally, the survey asked for rating of organizational aspects of the workshops including their organization, delivery, quality of feedback received and quality of the overall experience. These questions overlap with typical questions asked in other places such as the National Student Survey in UK. In addition, the final survey encouraged free comments both positive and critical to capture other issues not covered by the presented questions.

The method for assessing students' performance on the course was based on their scores in the workshop activities and a final exam. The questions for the final exam, which was taken at the end of the semester covered the workshop activities and lecture sessions. The final exam carried a total of $60 \%$ of the overall examination mark. Students' engagement and understanding of the learning activities were assessed by their performances in the hands-on lab exercises. The exercises were marked at the end of each workshop and carried a total of $40 \%$ of the overall examination mark. This is the model used for assessing workshop activities at the University of Ghana and we decided to maintain it to enable us make a fair comparison between students' performances in the pre-intervention

years where no robots were used and the intervention year where robots were introduced to conduct the learning activities.

\subsection{Participants}

The study was conducted for the first year Computer Science students at the Department of Computer Science, University of Ghana, during their first semester of study in Winter 2015. From a total of 202 students who enrolled on the course, 194 participated in at least one workshop session while 166 participated in all the workshops. 178 students participated in the initial survey and 166 participated in the final survey. The students were divided into 10 groups (roughly 20 students per each group). Because of the large size of the first year cohort and a limited number of robots (we only had 10 Thymio II robots available), the students were divided into pairs with one robot allocated to each pair. Although a 
limiting factor, this enabled us also to assess students abilities working in teams and observe their interactions during the learning activities.

Based on initial survey, we were able to assess the students' background. The majority of students declared their prior educational background in Science $(77 \%)$ with the remainder roughly split equally between Arts and Business. $19 \%$ of the students declared Computer Science as their major study subject and similar figures were reported both for minors in Maths and Stats (24\%) and Psychology (32\%). The reminder (13\%) accounted for minors in Maths and Economics. $22 \%$ of participants were female students which is a slightly higher figure than in the majority of the developed countries. Since this was the first year of study, it was not surprising that the majority of student (63\%) had no prior contact with programming or very basic understanding (25\%). Similarly, the students prior contact with robots was either through leisure activities (9\%) or basic programming $(9 \%)$ but the majority have not experienced any contact with such technology $(82 \%)$.

\section{Results}

The experimental data for the surveys and exam were statistically analyzed using SPSS for Windows version 21 to determine the effect the robotic activities had on students' learning. This was done by comparing the individual workshop survey results for the paper-based and robot-based activities, analysis of reports from the final survey and comparing the exam results from the pre-intervention and intervention years. In all comparison results, we report a significance value $p$ and assume that cases where $p<.05$ indicate statistically significant differences. We have highlighted all such entries in the tables to make visual comparisons easier. In addition, we also report a Pearson's correlation coefficient $r$ for paired final survey data on aspects of student learning to indicate the strength and direction of the linear relationship between two metrics ranging from -1 (perfect negative effect) to +1 (perfect positive effect) with 0 indicating no effect.

\subsection{Students' self-assessment of the activities}

An independent-samples t-test was conducted on students responses concerning the workshop activities to compare the means scores between the paper- and robotic-based activities. Table 2 presents detailed results from direct comparisons of each workshop activity. The table reports the workshop number, type of activity (paper- vs robot-based) and the student mean responses to three questions on workshop difficulty, relevance to concepts learnt in lectures and re-use of concepts learnt in previous sessions.

Since each group of students exercised the same topic twice, either as paperor robot-based activity, we have also made a clear distinction between groups which practiced the activities in the first or second week for a particular topic. Thanks to that we could see if some familiarity with the topic has any influence on the subjective perception of activities. 
Table 2: Student assessment of individual workshop activities. 'W' denotes workshop number, $1^{\text {st }}$ paper refers to paper-based activities taken in the first week and $2^{\text {nd }}$ paper in the second week for a particular topic. The robot-based activities are indicated in a similar fashion.

\begin{tabular}{|c|c|c|c|c|c|c|c|}
\hline \multirow{2}{*}{ W } & \multirow{2}{*}{ Activity } & \multicolumn{2}{|c|}{ Concepts employed } & \multicolumn{2}{|c|}{ Concepts re-used } & \multicolumn{2}{|c|}{ Difficulty } \\
\hline & & $\mu \pm \sigma$ & $\mathrm{p}$ & $\mu \pm \sigma$ & $\mathbf{p}$ & $\mu \pm \sigma$ & $\mathbf{p}$ \\
\hline \multirow{4}{*}{1} & $1^{\text {st }}$ paper & $2.9 \pm 1.2$ & \multirow{2}{*}{.57} & $2.8 \pm 1.2$ & \multirow{2}{*}{.00} & $2.4 \pm .8$ & \multirow{2}{*}{.06} \\
\hline & $1^{\text {st }}$ robot & $2.7 \pm 1.4$ & & $1.9 \pm 1.2$ & & $2.0 \pm .7$ & \\
\hline & $2^{\text {nd }}$ paper & $3.3 \pm 1.2$ & \multirow{2}{*}{.66} & $3.0 \pm 1.3$ & \multirow{2}{*}{.56} & $2.6 \pm 1.2$ & \multirow{2}{*}{.25} \\
\hline & $2^{\text {nd }}$ robot & $3.1 \pm 1.1$ & & $2.8 \pm 1.1$ & & $2.1 \pm 1.2$ & \\
\hline \multirow{4}{*}{2} & $1^{\text {st }}$ paper & $4.2 \pm .8$ & \multirow{2}{*}{.25} & $3.9 \pm 1.2$ & \multirow{2}{*}{.82} & $2.2 \pm .7$ & \multirow{2}{*}{.05} \\
\hline & $1^{s t}$ robot & $3.8 \pm 1.2$ & & $3.8 \pm 1.3$ & & $2.9 \pm 1.0$ & \\
\hline & $2^{\text {nd }}$ paper & $4.2 \pm 1.0$ & \multirow{2}{*}{.46} & $3.9 \pm 1.1$ & \multirow{2}{*}{.48} & $1.9 \pm 1.0$ & \multirow{2}{*}{.00} \\
\hline & $2^{\text {nd }}$ robot & $4.0 \pm 1.0$ & & $4.0 \pm 1.0$ & & $2.6 \pm 1.0$ & \\
\hline \multirow{4}{*}{3} & $1^{\text {st }}$ paper & $4.0 \pm 1.1$ & \multirow{2}{*}{.22} & $3.2 \pm 1.3$ & \multirow{2}{*}{.00} & $2.9 \pm .9$ & \multirow{2}{*}{.84} \\
\hline & $1^{\text {st }}$ robot & $4.2 \pm .9$ & & $4.0 \pm 1.0$ & & $2.9 \pm 1.0$ & \\
\hline & $2^{\text {nd }}$ paper & $4.1 \pm 1.0$ & \multirow{2}{*}{.48} & $3.9 \pm 1.1$ & \multirow{2}{*}{.68} & $2.4 \pm 1.0$ & \multirow{2}{*}{.00} \\
\hline & $2^{\text {nd }}$ robot & $4.0 \pm 1.1$ & & $4.0 \pm 1.1$ & & $3.0 \pm 1.2$ & \\
\hline \multirow{4}{*}{4} & $1^{\text {st }}$ paper & $3.1 \pm 1.1$ & \multirow{2}{*}{.25} & $3.2 \pm 1.2$ & \multirow{2}{*}{.01} & $3.6 \pm .8$ & \multirow{2}{*}{.10} \\
\hline & $1^{\text {st }}$ robot & $3.3 \pm 1.1$ & & $3.9 \pm 1.1$ & & $3.1 \pm 1.0$ & \\
\hline & $2^{\text {nd }}$ paper & $3.7 \pm 1.1$ & \multirow{2}{*}{.08} & $3.8 \pm 1.0$ & \multirow{2}{*}{.67} & $3.2 \pm .9$ & \multirow{2}{*}{.57} \\
\hline & $2^{\text {nd }}$ robot & $3.4 \pm 1.2$ & & $3.8 \pm 1.0$ & & $3.1 \pm 1.0$ & \\
\hline \multirow{4}{*}{5} & $1^{\text {st }}$ paper & $3.3 \pm 1.3$ & $0 ?$ & $3.1 \pm 1.1$ & 00 & $3.5 \pm 1.1$ & \\
\hline & $1^{\text {st }}$ robot & $3.9 \pm 1.1$ & .02 & $4.0 \pm 1.1$ & .00 & $2.9 \pm .9$ & .01 \\
\hline & $2^{\text {nd }}$ paper & $3.9 \pm .8$ & 13 & $3.8 \pm .8$ & 36 & $3.4 \pm 1.0$ & 00 \\
\hline & $2^{\text {nd }}$ robot & $3.7 \pm 1.1$ & & $3.6 \pm 1.0$ & .00 & $2.7 \pm 1.0$ & .00 \\
\hline
\end{tabular}

There were no major differences between paper- and robot-based activities when employing concepts learnt during lectures, with an exception of Workshop 5 on Control Structures, where robotic activities were perceived as more relevant to the lecture content. Overall, it can be seen that in this category activities prepared for Workshop 2 and 3 (Data Representation and Digital Logic) were considered as particularly useful indicated by higher mean scores and that students saw less relevance of lecture material corresponding to Workshop 1 (Introduction to Programming).

In gradual development of skills, indicated by a 'concepts re-used' question, the robot-based activities were perceived as more beneficial (see scores for Workshops 3, 4 and 5). The scores for Workshop 1 favor paper-based activities but that question was probably less relevant just at the beginning of the course and perhaps the students were not exactly sure what that particular question refereed to in that week.

There seems to be an increasing trend in the perceived difficulty of both types of workshop activities indicated by increasing mean scores for the difficulty 
question. Robotic tasks in Workshop 5 were perceived easier than paper-based assignments. There is an indication that paper-based activities for Workshop 2 and 3 might have been easier but since this is only reported by groups taking robotic tasks in the second week, these results are not entirely convincing.

Table 3 presents results obtained from the final survey on different aspects of learning for both paper-based and robot-based activities. In this overall comparison, the robot-based activities were more positively scored in categories of motivation and engagement, and also were perceived as easier as indicated by the mean scores. There was no difference in responses to questions on understanding and intuitiveness of both types of activities though.

Table 3: Students' self-assessment for their learning. Sample size $=166$.

\begin{tabular}{|c|c|c|c|c|}
\hline Metric & Activity & $\mu \pm \sigma$ & $p$ & $\mathbf{r}$ \\
\hline \multirow{2}{*}{ Motivating } & Paper-based & $3.7 \pm .8$ & \multirow{2}{*}{.00} & \multirow{2}{*}{.38} \\
\hline & Robot-based & $4.2 \pm .8$ & & \\
\hline \multirow{2}{*}{ Engaging } & Paper-based & $3.9 \pm .8$ & \multirow{2}{*}{.00} & \multirow{2}{*}{.33} \\
\hline & Robot-based & $4.2 \pm .7$ & & \\
\hline \multirow{2}{*}{ Understanding } & Paper-based & $4.1 \pm .8$ & \multirow{2}{*}{.75} & \multirow{2}{*}{.53} \\
\hline & Robot-based & $4.1 \pm .7$ & & \\
\hline \multirow{2}{*}{ Difficulty } & Paper-based & $3.1 \pm .9$ & \multirow{2}{*}{.02} & \multirow{2}{*}{.23} \\
\hline & Robot-based & $2.8 \pm 1.0$ & & \\
\hline \multirow{2}{*}{ Intuitive } & Paper-based & $3.6 \pm .8$ & \multirow{2}{*}{.83} & \multirow{2}{*}{.59} \\
\hline & Robot-based & $3.6 \pm .8$ & & \\
\hline
\end{tabular}

\subsection{Students' Exam Performance}

We have also compared the overall student exam results in the intervention year, where the robotic activities were introduced, to 5 pre-intervention years. The results presented in Table 4 indicate potential differences in Year 3 and 5 but overall there are no clear indications that the robotic activities impacted the marks achieved by students. It is worth to note that the course was taught by different lecturers in these years so the marks might be more correlated to the lecturing style rather than the type of activities. The natural variations in overall student academic abilities each year might also have influenced these results.

\section{Discussion}

In this work, we set out a goal of assessing the effectiveness and impact of robotic activities introduced into a regular higher education computer science curricula in a developing country. The presented results are promising especially in areas of student motivation and engagement. This was also evident in provided free 
Table 4: Comparison of pre-intervention and intervention years Exam results. * Year 6 is the intervention year.

\begin{tabular}{|l|c|c|c|}
\hline \multirow{2}{*}{ Year } & Sample Size & $\boldsymbol{\mu} \pm \boldsymbol{\sigma}$ & Comparison with Year 6 \\
\cline { 3 - 4 } & & & $\mathbf{p}$ \\
\hline 1 & 182 & $62.9 \pm 11.0$ & .11 \\
2 & 269 & $62.8 \pm 11.8$ & .08 \\
3 & 308 & $\mathbf{6 2 . 5} \pm \mathbf{1 1 . 6}$ &. $\mathbf{0 4}$ \\
4 & 373 & $65.2 \pm 10.0$ & .65 \\
5 & 209 & $\mathbf{7 0 . 1} \pm \mathbf{9 . 8}$ &. $\mathbf{0 0}$ \\
$1-5$ combined & 1341 & $64.6 \pm 11.2$ & .84 \\
\hline $\mathbf{6}$ & $\mathbf{1 6 6}$ & $\mathbf{6 4 . 8} \pm \mathbf{1 1 . 3}$ & \\
\hline
\end{tabular}

comments which include terms like practical, engaging, interactive, etc. The results from evaluation of individual workshops indicate that robotic activities seem to also have a positive effect on gradual development of skills and seeing links between different topics of the course.

In our case, it was more difficult to see the benefits of using educational robots for teaching specific theory topics and technical skills. This is a complex problem in which all components of the course delivery are playing a vital part, including lecture content, lecturing style but also quality and execution of the prepared practical activities. One particular problem we encountered is related to the ordering of topics in this particular course. As the robot exercises required learning some programming concepts from the very start, this introduced another level of complexity for students when they were just learning fundamentals of computer architectures. It would be interesting to see if a course which would start with programming principles first would be more effectively delivered. The analysis of student performance in pre- and intervention years suggest that using overall student marks from the course as an effectiveness indicator is of very limited use and methodological developments in evaluating similar activities should focus on other methods (e.g. short tests after each activity).

Another purpose of our study was to evaluate the effectiveness of using robotic set-up as a tool for conducting educational activities. The results of the study indicate that our robotic set-up was generally effective. For example, students' attendance to the lab sessions improved as the result of the introduction of the robot-based activities. Also students were able to perform different educational tasks with the robots which made their learning meaningful. This was noticeable from some of the comments students made concerning the robotbased activities. Statements like "it was very understandable and interesting", "it was interactive and helpful", "it opened my mind to different ways of approaching a problem" were made in the self-assessment report. The selected hardware platform, Thymio II robot proved to be a reliable choice. Its rich functionality enabled us to introduce a wide range of activities and programming concepts. There were some limitations in illustrating specific technical topics (e.g. binary to decimal conversions) due to platform limitations but nevertheless 
from the point of view of an educator this was a great platform to work with. The provided teaching materials and example projects helped our students to understand some of the programming concepts.

The main problem we encountered in delivering these activities were outside of typical spectrum of educational challenges. Frequent power outages disrupted the scheduled sessions and might have had a negative impact on student perception of the course. Such infrastructure problems are not only limited to a single developing country but more prevalent across this part of the world. One way of solving this problem might be to consider environments relying on mobile devices only, which require less power resources and can be powered from batteries. Also due to the limited number of robots we used for the study, students could only practice their coding skills in the labs where the robots were kept. Students complained about this situation and expressed the desire to own the robots so that they can work on their assignments at the halls of residence as well. Some of these problems might be alleviated by introduction of simulators which could be used outside of the scheduled activities.

\section{Conclusions}

In this work, we reported on our experience in integrating educational robotics activities into higher education curricula in developing countries. In particular, we investigated methodology for assessing the effectiveness and impact of such activities on student engagement and learning. In future work, we would like to look closer at potential learning gains when using educational robots for teaching specific topics in Computer Science. We would also like to investigate how the effectiveness of such activities changes in longer term. Our results indicate some positive benefits gained from such activities and indicate that educational robotics is a promising tool in developing engaging study curricula. We hope that our experience from this study together with the provided materials resulting from this study will be beneficial to other educators working with educational robotics in different parts of the world.

\section{References}

1. Cielniak, G., Bellotto, N. and Duckett, T.: Integrating mobile robotics and vision with undergraduate computer science. IEEE Transactions on Education, 56(1), pp.48-53 (2013)

2. Mills-Tettey, G. A., Dias, M. B., Browning, B., and Amanquah, N.: Teaching technical creativity through Robotics: A case study in Ghana. In: Workshop on AI in ICT for Development, Int Conf. on AI (2007)

3. Gyimah, E.K.: Teachers use of instructional strategies in primary schools in Ghana: implication to inclusive education. Education Research Journal, 1(3), pp.46-52 (2011)

4. Onsongo, J.: Affirmative action, gender equity and university admissions-Kenya, Uganda and Tanzania. London Review of Education, 7(1), pp.71-81 (2009) 
5. Paras, J.: Crisis in mathematics education. Student failure: Challenges and possibilities. South African Journal of Higher Education, 15(3), pp.66-73 (2001)

6. Yerion, K.A. and Rinehart, J.A.: Guidelines for collaborative learning in computer science. ACM SIGCSE Bulletin, 27(4), pp.29-34 (1995)

7. Akey, T.M.: School Context, Student Attitudes and Behavior, and Academic Achievement: An Exploratory Analysis. MDRC (2006)

8. Papert, S.: The children's machine. Technology Review-Manchester NH-, 96, 28-28 (1993)

9. Nourbakhsh, I. R., Crowley, K., Bhave, A., Hamner, E., Hsiu, T., Perez-Bergquist, A., and Wilkinson, K.: The robotic autonomy mobile robotics course: Robot design, curriculum design and educational assessment. Autonomous Robots, 18(1), 103-127 (2005)

10. Alimisis, D.: Robotics in education \& education in robotics: Shifting focus from technology to pedagogy. In Proceedings of the 3rd International Conference on Robotics in Education pp. 7-14 (2012)

11. Frangou, S., Papanikolaou, K., Aravecchia, L., Montel, L., Ionita, S., Arlegui, J., \& Monfalcon, S.: Representative examples of implementing educational robotics in school based on the constructivist approach. In SIMPAR Workshop on Teaching with robotics: didactic approaches and experiences, Venice, Italy (2008)

12. Barker, B. S., and Ansorge, J.: Robotics as means to increase achievement scores in an informal learning environment. Journal of Research on Technology in Education, 39(3), 229-243 (2007)

13. Rogers, C., and Portsmore, M.: Bringing engineering to elementary school. Journal of STEM Education: innovations and research, 5(3/4), 17 (2004)

14. Touretzky, D.S.: Robotics for computer scientists: whats the big idea?. Computer Science Education, 23(4), pp.349-367 (2013)

15. Souza, M.A. and Duarte, J.R.: Low-cost educational robotics applied to physics teaching in Brazil. Physics Education, 50(4), p.482 (2015)

16. Becker, B.W.: Teaching CS1 with karel the robot in Java. In ACM SIGCSE Bulletin (Vol. 33, No. 1, pp. 50-54). ACM (2001)

17. Gyebi, E., Hanheide, M., and Cielniak, G.: Educational robotics for teaching computer science in Africa-pilot study. In Int. Workshop on Educational Robots (2015)

18. McNamee, L., Roberts, T., and Williams, S.: Online collaborative learning in higher education (2001)

19. Gyebi, E., Hanheide, M., Cielniak, G.: Affordable mobile robotic platforms for teaching computer science at African universities. In Int. Conf. on Robotics in Education, (2015) 